\title{
Two Dimensional Numerical Models of the California Electromagnetic Coastal Anomaly
}

\author{
C.S. Cox and J.H. Filloux \\ Scripps Institution of Oceanography, La Jolla, Ca., U.S.A.
}

(Received October 23, 1973; Revised April 13, 1974)

Two dimensional numerical models of the California coast anomaly have been constructed to compare their predictions with observations, with a view to infer the electrical conductivity structure of crust and upper mantle within this area. The third axis of these models is directed along the coast. Only induction processes with magnetic fluctuations normal to it are considered (Transverse Electric Mode). The ocean is represented by a this sheet with a conductivity equal to the vertically integrated oceanic conductivity. Crust and upper mantle are simulated by free and finite conductivity distributions extending downward several skin depths before termination by an infinitely conductting horizontal plane. The upper boundary, which contains the source field, is transparent to the upward reflected energy, thus simulating the high impedance of the ionosphere. The horizontal wave length of the source fields is long, though arbitrary, and these fields can simulate horizontal perturbations, stationary or progressive. The predicted fields are obtained by solving for the stream function $\psi$ of the magnetic fluctuation vector $\boldsymbol{B}$ over a rectangular area centered around the coast. $\psi$ has to satisfy the diffusion equation $\nabla^{2} \psi+$ $i \mu \mu_{0} \omega \sigma \psi=0$ subject to the aforementioned boundary and interface conditions. Application of these techniques to interpret observations of electromagnetic fluctuations in the range $2^{-2}$ to $2^{1} \mathrm{cph}$ across the central California coast suggests a substantial increase of conductivity of the basement, seaward of the continental slope.

PARKinson $(1959,1962)$ observed that along almost all oceanic margins the lines of magnetic variation dip steeply inshore in the vicinity of the coast. This widespread coastal effect is explained by refraction of the induced currents within an anisotropic distribution of electrical conductivity across the shoreline, higher conductivity occurring offshore. Thus electric currents concentrate along the coast and seaward of it. An obvious contribution to this feature is the high conductivity of the ocean. However, the dissimilarities between oceanic and continental basement suggested by various geophysical investigations may also reflect an appropriate conductivity contrast in that area (Raitt, 1963; Ewing, 1963; Shor and Raitt, 1967; Oliver and Dorman, 1963; Worzel, 1963).

From an extensive survey of the prominent coastal anomaly of central 
California by means of magnetometer arrays deployed inshore, ScHMUCKER (1964) has shown that offshore data are also required to ascertain whether this effect originates from the ocean alone or whether crustal and upper mantle conductivity inhomogeneities are also involved. From measurements of the electric field at several locations in the adjacent ocean as well as of the total magnetic variation at the surface near the base of the continental slope where electric currents are most intense we have concluded (FILloux, 1967; Cox et al., 1971) that the electric currents induced in the ocean account for part only of the observed California coastal effect. This conclusion has been reached by comparing observations to the predictions of mathematical models of the coastal anomaly for an appropriate range of their variable parameters. Inshore and offshore optimum conductivity distributions have been obtained and their transition has been found to occur near the continental slope. Our purpose is to describe these models.

The generalized two-dimensional coastal effect results from the superposition of two uncoupled modes generally referred to as transverse electric, TE, and transverse magnetic TM. In the TE mode (see Fig. 1) electric field and electric current are everywhere horizontal, parallel to the coast and constant along it ( $y$ azis). Thus, they have no $x, z$ components. The magnetic variation is normal to the coast and has no $x$ component. The TM mode (see Fig. 1) for which the magnetic variation is parallel to the coast has no conspicuous magnetic signature. It is more readily identified by the strong intensification inland of the electric field normal to the coast. Thus, the prominent coastal effect discovered by Parkinson is the expression of transverse electric induction processes. The TE mode is the only one considered in the following.

The specific features of the models are sketched on Fig. 2. Ionospheric currents flowing in the thin and little conducting ionosphere in a direction parallel to the coast generate horizontal electromagnetic perturbations in the TE mode. Part of their energy propagates downward, unattenuated through the atmosphere, then diffuses through ocean and earth, partly absorbed and
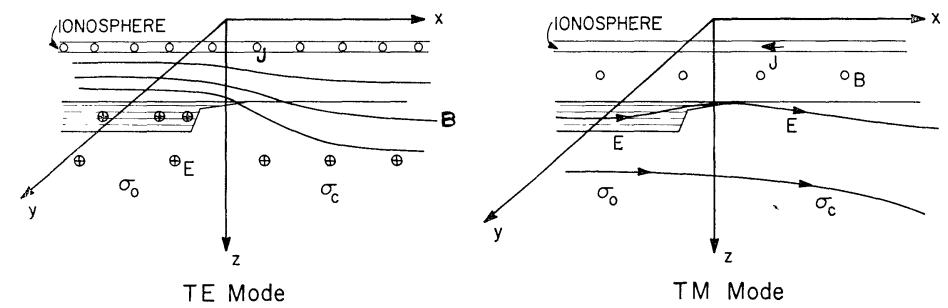

Fig. 1. Transverse electric and transverse magnetic modes of the low frequency electromagnetic coastal effect. The TE mode is most conspicuous as evidenced by the sharp increase of the vertical magnetic fluctuations near the coast. The TM mode produces a sharp enhancement of the electric field normal to the coast, in the absence of pronounced magnetic effect. 


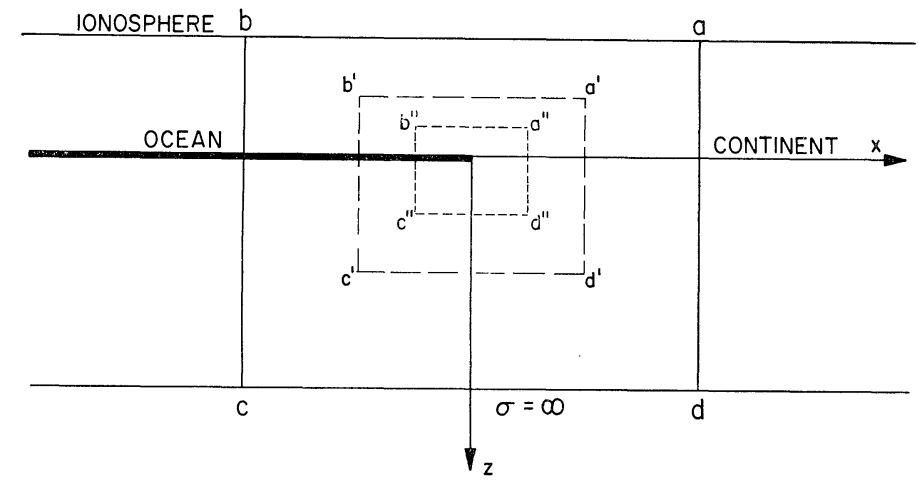

Fig. 2. Diagram of the assumed two-dimensional models of the TE coastal effect. The ionospheric currents are assumed to be contained in a thin, horizontal layer of low electrical conductivity and to be smoothly varying horizontally. The ocean is assumed to be inifinitely thin with a conductivity equal to the vertically integrated conductivity of sea water and sediments. To the right and to the left the continental and oceanic conductivity structures are horizontally uniform. Along the side boundaries $a-d$ and $b-c$ the coastal effect is assumed to have decayed to a negligible level, and along the lower boundary $\mathrm{c}-\mathrm{d}$ the residual fluctuation are assumed to be neglibibly small.

partly reflected. The reflected wave, distorted by the conductivity inhomogeneities encountered, escapes freely upward through the ionosphere which is too little conducting at the frequencies of interest to allow secondary reflection $\left(\mu_{0} \omega \sigma_{i} h_{i} \ll 1, \sigma_{i}\right.$ and $h_{i}$ conductivity and thickness of ionosphere, $\mu_{0}$ permeability, $\omega$ angular speed). A lateral dependence of the form $\mathrm{e}^{-i(\omega t \pm k x)}$ simulates perturbations of wavelength $2 \pi k^{-1}$ moving along $x$ with the velocity $\omega k^{-1}$ ( $k=$ wave number). Appropriate summation of individual perturbations of this form permits the simulation of a variety of real situations. For instance, a single wave represents a perturbation moving offshore $(+\operatorname{sign})$ or inshore $(-\operatorname{sign})$, a case representative of the east-west sweep of the daily solar variation. The superposition of two equal and oppositely running waves represents stationary perturbations with horizontal uniformity adjustable by varying their relative phase. Magnetic storm perturbations are more consistent with such representation. In all cases, the observed widespread uniformity of the ionospheric impulses suggests that the use of a single and small $k$ value is adequate, providing the corresponding wave length $2 \pi k^{-1}$ is several thousand $\mathrm{km}$ long.

At the center of the model, the rectangular area a, b, c, d (see Fig. 2) encloses the region of interest, that is not only the region over which conductivity homogeneities occur, but also within which their electromagnetic signature remains appreciable. The conductivity structures to the right and to the left are independently uniform horizontally to infinity, one representing a continental conductivity profile, the other an oceanic type. 
At a sufficient depth below the earth's surface the diffusing perturbation attenuates to a negligible level and the model can be terminated there.

Classic electromagnetic theory demonstrates that penetration of electromagnetic energy into conducting materials follows the diffusion equation. If $\phi(x, z, t)$ is the stream function of the solenoidal magnetic vector $\boldsymbol{B}$, and if an harmonic time dependence of the form $\phi \rightarrow \psi \mathrm{e}^{-i \omega t}$ is implied, then $\psi$ satisfies everywhere the relation

$$
\nabla^{2} \psi+i a \psi=0
$$

where $i=(-1)^{1 / 2}, a=\mu_{0} \mu \sigma \omega\left(\mu_{0}\right.$ permeability in a vacuum, $\mu$ relative permeability of the medium, $\sigma$ electrical conductivity. $\mu$ is taken equal to unity everywhere).

The magnetic field components $H$ along $x$ and $Z$ along $z$, and the electric field $E$ along $y$, then satisfy

$$
H=\frac{\partial \psi}{\partial z}, \quad Z=-\frac{\partial \phi}{\partial x}, \quad E=-i \omega \phi
$$

Our objective is thus to find a function $\phi$ satisfying (1) over the useful area $\mathrm{a}, \mathrm{b}, \mathrm{c}, \mathrm{d}$ and subject to boundary conditions that represent correctly the model features described earlier.

The vanishing of $H$ and $Z$ at great depth requires $\psi$ to be constant there, while the vanishing of $E$ requires $\phi$ to be zero, see (2). Thus, the required condition along the lower boundary is $\psi=0$.

Far to the right and to the left where the conductivity is uniform horizontally and where the coastal effect is not felt anymore, the $x$ and $y$ dependence of $\phi$ can be separated according to

where $M$ and $N$ satisfy

$$
\phi(x, z)=M(x) N(z)
$$

$$
\begin{aligned}
& \frac{\partial^{2} M}{\partial x^{2}}+k^{2} M=0 \\
& \frac{\partial^{2} N}{\partial z^{2}}+\kappa N=0
\end{aligned}
$$

with

$$
\kappa=k^{2}-i a
$$

Thus, $M$ and $N$ have solutions of the form

$$
\begin{aligned}
& M=C_{1} \mathrm{e}^{-i k x}+C_{2} \mathrm{e}^{i k x} \\
& N=C_{3} \mathrm{e}^{-i \kappa z}+C_{4} \mathrm{e}^{i \kappa z}
\end{aligned}
$$

$C_{1}$ and $C_{2}$ are constants dependent, as seen earlier, upon the type of ionospheric perturbation under consideration. $C_{3}$ and $C_{4}$ are fixed constants within individual layers of uniform conductivity. They are, therefore, dependent not only 
upon $C_{1}$ and $C_{2}$ but also upon layer, thus upon depth. In otherwords, $N$ to the right and to the left are piecewise analytic functions that can be found by classic methods (CAGNIARD, 1953; WaIT, 1962). In stratified systems with conductivity varying continuously with depth, appropriate discretization of the conductivity profile permits $N$ to be approximated to the degree needed. When $M$ and $N$ have been found the solution far to the right (r) and to the left (1) is of the form

$$
\phi_{\mathrm{r}}=C_{\mathrm{r}} M(x) N_{\mathrm{r}}(z), \quad \phi_{1}=C_{1} M(x) N_{1}(z)
$$

where $C_{\mathrm{r}}$ and $C_{1}$ are complex constants which, when known, determine the boundary values for the right and left sides of the area of interest $a, b, c, d$. However, appropriate scaling of $C_{\mathrm{r}}$ and $C_{1}$ requires careful treatment of the upper boundary condition.

In the highly inhomogeneous coastal area, the induced fields do not conserve the horizontal uniformity of the ionospheric currents. The reflected perturbation, on the contrary, has acquired energy within a continuum of wave lengths shorter than that of the incident perturbation $2 \pi k^{-1}$. Because of the highly resistive and little inductive nature of the ionosphere, the reflected waves escape freely into outer space. From the standpoint of an observer on the earth's surface interested in the earth's interior, the observable electromagnetic effects are made of two independent contributions, namely an external one (upperscript e) with energy travelling downward, and an internal one (upperscript i) with energy travelling upward. Hence, the stream function $\psi$ results from the superposition of an external part $\psi^{\text {e }}$ associated with the ionospheric currents and an internal part $\psi^{\mathrm{i}}$ associated with the eddy currents induced in oceans and earth. Clearly, the appropriate upper boundary condition for our models requires horizontal uniformity of the external field only, thus of $\psi^{\mathrm{e}}$ alone.

Far to the right and to the left where conditions are uniform, the ratios $R_{\mathrm{r}}$ and $R_{1}$ of $\psi^{e}$ to $\phi$ can be found by classic methods as indicated earlier. Since $\psi^{\mathrm{e}}$ must be the same function over the entire model, from right to left, then $C_{\mathrm{r}}$ and $C_{1}$ in (8) must satisfy

$$
R_{\mathrm{r}} C_{\mathrm{r}} N_{\mathrm{r}}\left(z=z_{\text {ionosphere }}\right)=R_{1} C_{1} N_{1}\left(z=z_{\text {ionosphere }}\right) .
$$

Normalizing, for convenience, all values with respect to $\psi=1$ at the upper right corner, a, of the model area determines all constants and fixes the lateral boundaries.

The function $\psi$ cannot be stipulated at once along the upper boundary of the rectangle a, b, c, d since $\psi^{\mathrm{e}}$ only is known here. Our scheme will be to impose the proper constraint in successive steps.

At first $\psi$ is replaced by $\phi^{e}$. This leads to discontinuities of $\psi$ and its derivatives at the model corners $a$ and $b$. The latter are artificially removed by blending the function representing $\psi$ over the central model area with those representing $\psi$ on the right and left sides. This is achieved by fading one in, while fading 
the other out. For this purpose complementary cosine functions acting upon one quarter of the central model area have been used.

When a solution $\psi_{n}$ is found for the above situation ( $n=1$ at first), its internal contribution $\psi_{n}^{\mathrm{i}}$ at the upper boundary is calculated (see Appendix) and an improved upper boundary condition is obtained by replacing there $\phi=\psi^{\mathrm{e}}$ by $\phi=\psi^{e}+\psi^{\mathrm{i}}$. The operation is repeated until adequate convergence has occurred, a result rapidly achieved.

The depth of the ocean being small compared to the skin depth of sea water for the periods of interest, its representation as a thin conducting sheet with a conductivity $\sigma_{\text {oc }}$ equal to the vertically integrated conductivity of sea water and sediments is sufficiently faithful. In this case the appropriate interface condition is obtained by integrating (1) vertically to give

$$
\left(\frac{\partial \psi}{\partial z}\right)^{\text {above }}-\left(\frac{\partial \psi}{\partial z}\right)^{\text {below }}=-i \sigma_{\mathrm{oc}} \omega \mu_{0} \psi
$$

Exact analytic solutions for $\phi$ over the main model area a, b, c, d (Fig. 2) can be found only for highly idealized situations. Alternately an adequate approximation $\Psi_{(P)}$ of $\psi_{(x, z)}$ at a finite number of points $P$ over the area a, b, c, d can be obtained by appropriate discretization of (1) and solving numerically for $\Psi_{(P)}$ by means of high speed digital computer. Many aspects of this technique are discussed in Forsythe and Wasow (1960). We outline the procedure.

Using the square network of Fig. 3, the five-point discretization of (1) at $P$ becomes

$$
\frac{1}{4}\left(\Psi_{\mathrm{N}}+\Psi_{\mathrm{E}}+\Psi_{\mathrm{S}}+\Psi_{\mathrm{W}}\right)-\left(1-\lambda^{2} \frac{i}{4} a\right) \Psi_{P}=0
$$

where $\lambda$ is the mesh spacing and the subscripts $N$, E, etc... for north, east, etc... symbolize position relationships. The oceanic interface condition derived from (10) is simply

$$
\frac{1}{2}\left(\Psi_{\mathrm{N}}+\Psi_{\mathrm{S}}\right)-\left(1-i \sigma_{\mathrm{oc}} \frac{\omega}{2} \mu_{0} \lambda\right) \Psi_{(P)}=0
$$

In order to limit the discretization error in $H$ and $Z$ associated with the curvature of $\phi$ the mesh size $\lambda$ must be sufficiently small with respect to the local skin depth, $d=\left(\sigma \omega \mu_{0}\right)^{-1 / 2}$. Thus, the relation

a.

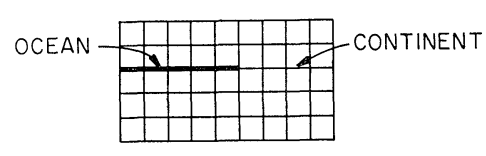

b.

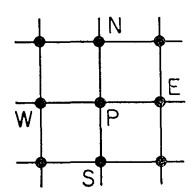

Fig. 3. (a) Diagram of the square grid model network. (b) Identification of the position of the grid points. 


$$
\sigma \omega \mu \lambda^{2} \ll 1
$$

must be satisfied for all values of $\sigma$. The choice $\sigma \omega \mu_{0} \lambda^{2} \leq 10^{-1}$ has been found adequate.

In order to insure the validity of $\phi=0$ along the lower boundary the distance of the latter from the earth's surface must be large enough to make residual fields at that level negligible. The following conditition

$$
\sum_{n}\left(\sigma_{n} \omega \mu_{0}\right)^{-1 / 2} \lambda^{-1} \geq 3
$$

where $\sum_{n}$ refers to a summation from earth's surface to lower boundary, insures that the residual fields at the lower boundary have fallen to less than $5 \%$ of their values at the earth's surface.

To allow frequencies and conductivities as high as 4 cycles per hour and $0.4 \mathrm{mho} \cdot \mathrm{m}^{-1}$ respectively may require a mesh size as small as $10 \mathrm{~km}$. Typically a strong coastal anomaly decays away from the coast to a negligible level at distances of several hundred kilometers. Thus, our square network has been chosen to extend 80 mesh distances horizontally. Allowing for 40 meshes on the vertical permits one to accommodate all realistic situations.

When (11) or (12) have been written for all grid points within the model area a system of linear equations

$$
g \Psi=s
$$

is generated, where $g$ is the sparse matrix of the coefficients, $\Psi$ the column matrix of the unknown $s$ and $s$ the column matrix representing the constraints imposed by boundary and interface conditions. If $U$ and $V$ are the number of grid columns and rows, including boundaries, the number of equations then equals $(U-2)(V-2)$ and, in the above case, is near 3200 .

Although methods exist today to invert $g$ directly, our solution scheme involved an over-relaxation approach described briefly in the following. Splitting the matrix $g$ into diagonal, subdiagonal and upper diagonal matrices $a, b$, and $c$, and symbolizing the over-relaxation approach by the iteration number $j$ and by the over-relaxation parameter $\Omega,(15)$ becomes

$$
\left(a+\Omega^{-1} b\right) \Psi_{j}+\left(c+\left(1-\Omega^{-1}\right)\right) b \Psi_{j-1}=s
$$

(see Young-Frankel theory in Forsythe and Wasow, 1960). Equation (16) indicates that the new values of $\Psi$ are obtained in part from new, and in part from previous values of $\Psi$. When $\Omega=1$ the scheme corresponds to a simple relaxation method. Increasing $\Omega$ from this value increases the speed of convergence until an optimum value is attained beyond which divergence sets in rapidly. An increase of convergence rate is usually associated with higher instability which in practice is not always readily distinguished from divergence. Thus, 
finding $\Omega_{\text {opt }}$ is a problem in itself. The advantage of finding it results from the fact that it does not vary rapidly with the models' parameters.

In the present case where the variable is complex and where the diffusion equation is involved, an approach to the study of the behavior of $\Omega_{\mathrm{opt}}$ modeled after that of Garabedian for Laplace's equation (Forsythe and WAsow, 1960) has shown that $\Omega_{\text {opt }}$ is complex, with a small negative imaginary part (FILloux, 1967). However, only minimal help was derived from this attempt, and $\Omega_{\text {opt }}$ was approached mainly by trial and error. Nevertheless, when direct inversion of large-size matrices is not possible, over-relaxation offers an alternate approach to the solution of a large system of linear equations. In our case, 100 to 300 iterations were required per model depending upon our success in closing in on $\Omega$ optimum.

We have seen earlier that the upper boundary conditions also depended upon successive improvements upon an initial set up. In practice, the successive corrections were carried out simultaneously with the over-relaxation iterations, but only ten times less frequently.

In order to insure that the lateral boundaries of the central rectangular model area be sufficiently far removed from the coastal conductivity features we have used a coarse initial grid mesh varying between 10 and $40 \mathrm{~km}$, depending on frequency and on maximum conductivity. However, the resulting wide spacing of the $\Psi_{P}$ values leads to insufficient detail in the spacial variation of the field components. This limitation was overcome by halving the mesh, yet keeping the same number of grid points, and maintaining the features of interest well centered. On Fig. 2 the successive reduced model areas are represented by the rectangles $a^{\prime}, b^{\prime}, c^{\prime}, d^{\prime}$, etc. The boundary conditions in this case are obtained by taking the computed value of $\Psi$ along the appropriate rows and columns, adding intermediate values at the new points by interpolation. When the final grid mesh falls to about $5 \mathrm{~km}$ a resolution sufficiently sharp of the predicted coastal effect is achieved.

Restricting the number of free parameters to the minimum, the main features of the central California coastal effect are found to be approached best by models for which the continental conductivity is negligible down to $30 \mathrm{~km}$, then rises downward at a rate near $2.7 \times 10^{-7} \mathrm{mho} \cdot \mathrm{m}^{-2}$, and the offshore conductivity is negligible within a layer a few $\mathrm{km}$ thick below the oceanic sediments, then rises at a rate near $1.5 \times 10^{-5} \mathrm{mho} \cdot \mathrm{m}^{-2}$. The transition between the two structures must be sharp and centered around the continental slope. These results have been presented elsewhere (Filloux, 1967; Cox et al., 1971).

The fit between observations and optimum model predictions can be examined on Fig. 4 for the frequencies $0.25,0.5,1.0$ and 2.0 cycles per hour. In opposition to idealized models using infinitely conducting planes which much be changed in function of the frequency, here the conductivity distribution remains unchanged for all frequencies tested. This constitutes a very stringent 

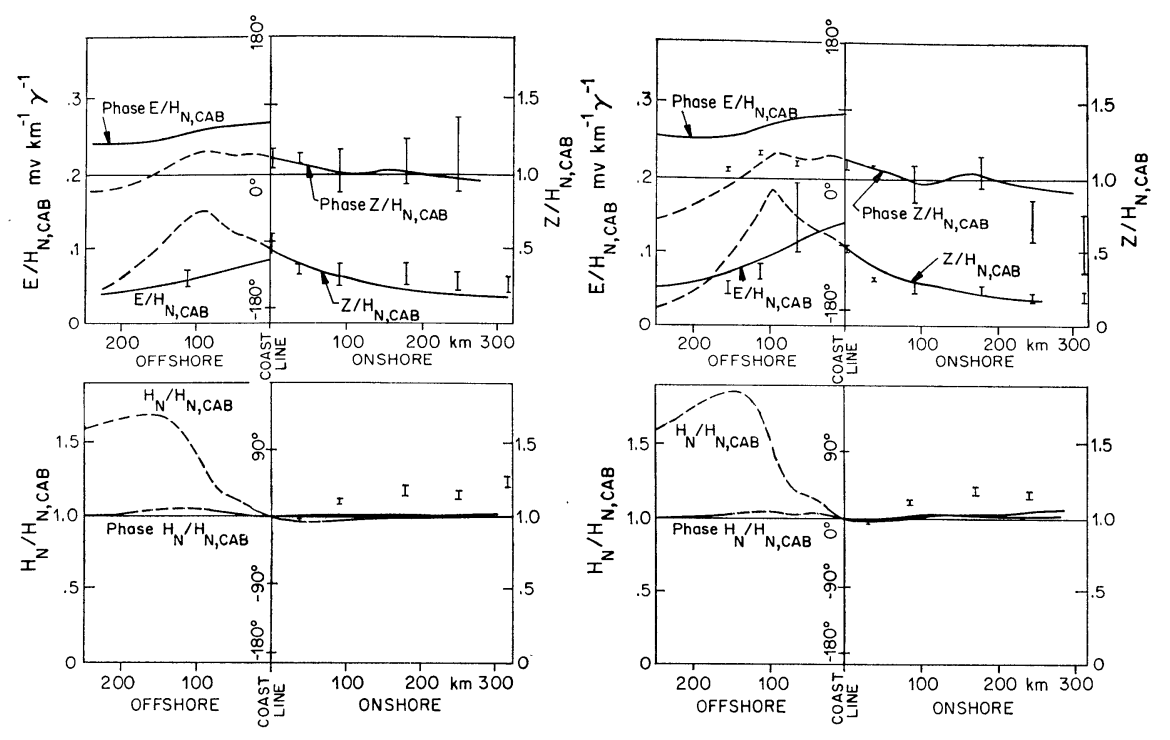

$f=.25 \mathrm{cph}$

$f=.5 \mathrm{cph}$
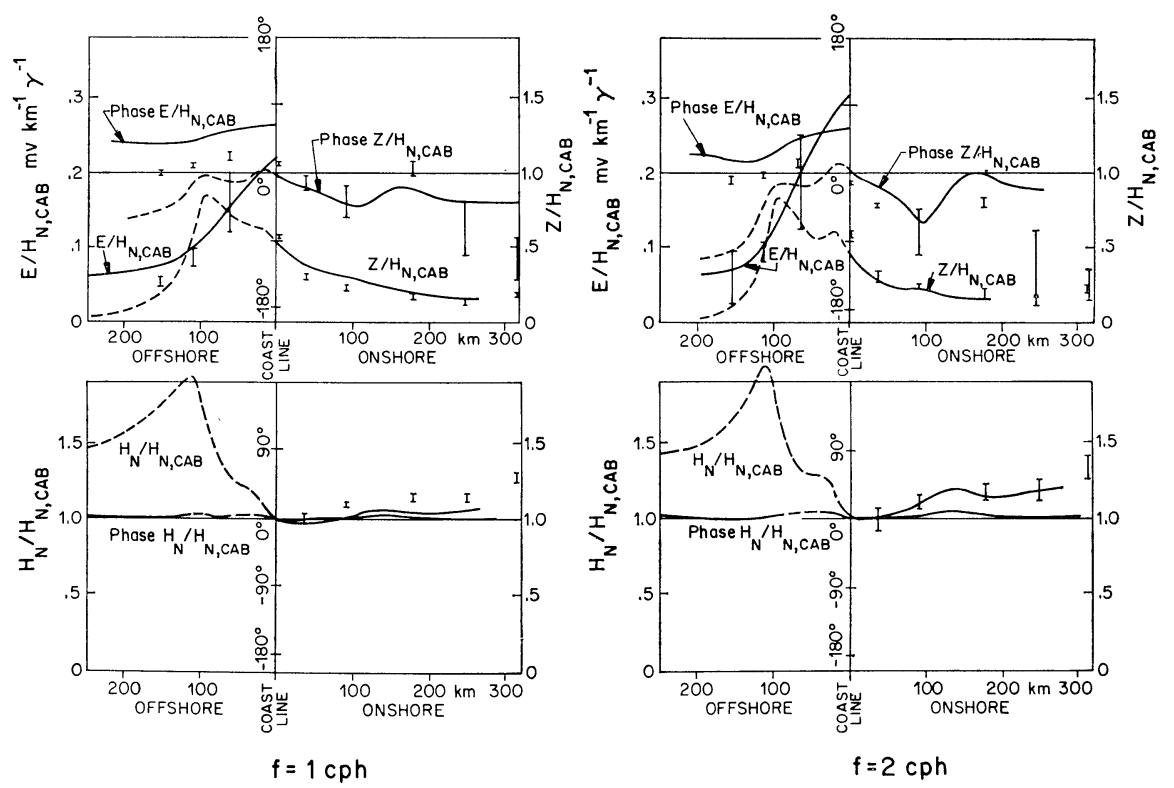

Fig. 4. Fit between observations and model predictions for frequencies $0.25,0.5,1.0$ and 2.0 cycles per hour and for the following conductivity structure: inshore from the continental slope $\sigma=0$ down to $30 \mathrm{~km}$, then increase linearly with depth at the rate of $0.0000027 \mathrm{mho} \cdot \mathrm{m}^{-2}$; offshore $\sigma=0$ down to $2 \mathrm{~km}$, then increases at the rate of $0.00015 \mathrm{mho} \cdot \mathrm{m}^{-2}$. The observed values are represented with their error bars. The computed field components are represented by lines, continuous where data exist and dashed where no data are available. 
requirement. In spite of this, a better fit of the data could be achieved with these direct conductivity models rather than with the other type. The remaining divergences between model and data results most probably from: (1) departure from true two-dimensionality of the coastal structure; (2) finite size of the ocean implying that the coast does not, in fact, extend to infinity in the $y$ direction; (3) neglecting the earth's curvature; and (4) remote influences of distant sources.

\section{Appendix. Upper Boundary Condition}

For simplicity $z=0$ along the upper boundary, and a complex notation is used to represent the total field $B, B=H+i Z$. The subscript $0, B_{0}=H_{0}+i Z_{0}$ refers to $x=0$.

Just below the ionosphere where the conductivity is negligible the stream function $\phi$, as well as its external and internal parts, satisfy Laplace's equation $\nabla^{2} \psi=0$. They may, thus, be represented by a sum of terms of the form $a(k) \mathrm{e}^{k(z+i x)}$. The external stream function $\phi^{\mathrm{e}}$, is characterized by a vertical dependence $-k z$ and $k z$ for positive or negative wave numbers $k$, respectively. The opposite is true for $\phi^{\mathrm{i}}$, thus

$$
\psi^{\mathrm{e}(\mathrm{or} \mathrm{i})}=\int_{-\infty}^{+\infty} k^{-1} b_{(k)}^{\mathrm{e}(\text { or i })} \mathrm{e}^{-(\text {or }+)|k| z+i k x} \mathrm{~d} k
$$

where the coefficient $b^{\mathrm{i}}$ is to be found (A.5).

The internal and external complex fields, which satisfy (2), thus are

$$
H_{0}^{\text {e(or i) }} \pm i Z_{0}^{\text {e(or i) }}=\int_{-\infty}^{+\infty}(-(\text { or }+) \operatorname{sign} k \pm 1) b_{(k)}^{\text {e(or i) }} \mathrm{e}^{i k x} \mathrm{~d} k
$$

The complex total field is then given by

$$
H_{0} \pm i Z_{0}=\int_{-\infty}^{+\infty}\left[\operatorname{sign} k\left(-b^{\mathrm{e}}+b^{\mathrm{i}}\right) \pm\left(b^{\mathrm{e}}+b^{\mathrm{i}}\right)\right] \mathrm{e}^{i k x} \mathrm{~d} k
$$

The Fourier inverse of (A.3) gives

$$
\operatorname{sign} k\left(-b^{\mathrm{e}}+b^{\mathrm{i}}\right) \pm\left(b^{\mathrm{e}}+b^{\mathrm{i}}\right)=\frac{1}{2 \pi} \int_{-\infty}^{\infty}\left(H_{0} \pm i Z_{0}\right) \mathrm{e}^{i k x} \mathrm{~d} x
$$

from which the required coefficients $b_{(k)}^{\mathrm{i}}$ are obtained according to

$$
b_{(k)}^{\mathrm{i}}= \pm \frac{1}{4 \pi} \int_{-\infty}^{+\infty}\left(H_{0} \pm i Z_{0}\right) \mathrm{e}^{i k x} \mathrm{~d} x, \quad+\text { for } k>0, \quad-\text { for } k<0
$$

In practice the Fourier integral of (A.5) can be replaced by a Fourier series extending sufficiently far away from the lateral boundary of the central model area.

This work was supported by the Office of Naval Research, the National Science Foundation and the American Petroleum Institute. 


\section{REFERENCES}

Cagniard, L., Principes de la methode magnetotellurique, Annales de Geophysique, 9, 95-125, 1953.

Cox, C.S., J.H. Filloux, and J.C. LARson, Electromagnetic studies of ocean current and electrical conductivity below the ocean floor, in "The Sea," edited by A. E. Maxwell, vol. 4, part 1, John Wiley and Sons, New York, 1971.

Ewing, J.I., The mantle rocks, in "The Sea," vol. 3, section 1, chapter 7, pp. 103-107, John Wiley and Sons, New York, 1963.

Filloux, J.H., Oceanic electric currents, geomagnetic variations and the deep electrical conductivity structure of the ocean-continent transition of central California. Thesis, University of California, San Diego, 1967.

Forsythe, G.E. and W.R. WASOw, Finite Difference Methods for Practical Differential Equations, John Wiley and Sons, New York, 1960.

Olivier, J. and J. Dorman, Exploration of sub-oceanic structure by the use of seismic surface waves, in "The Sea," edited by A. E. Maxwell, vol. 3, section 1, chapter 8, pp. 110-126, John Wiley and Sons, New York, 1963.

Parkinson, W.D., Direction of rapid geomagnetic fluctuations, Geophys. J. Roy. Astr. Soc., 2 , 1-14, 1959.

PARKSON, W.D., The influence of continents and oceans on geomagnetic variations, Geophys. J. Roy. Astr. Soc., 6, 441-449, 1962.

RAItT, R.W., The crustal rocks, in "The Sea," edited by A. E. Maxwell, vol. 3, section 1, pp. 85-101, chapter 6, John Wiley and Sons, New York, 1963.

Schmucker, U., Anomalies of geomagnetic variations in the southwestern United States, $J$. Geomag. Geoelectr., 15, 193-221, 1964.

Shor, G.G. and R.W. RAITT, Explosion seismic refraction studies on the crust and upper mantle in the Pacific and Indian Ocean, MPL-U-39/67, Univ. of California, 1967.

WAIT, J.R., Electromagnetic Waves in Stratified Media, MacMillan, New York, 1962.

Worzel, J., Gravity at sea, in "The Sea," vol. 3, section 1, chapter 9, edited by A. E. Maxwell pp. 134-154, John Wiley and Sons, New York, 1963. 